\title{
A Novel Approach to Assimilate VANET Communication using (WAVE) Wireless Access Vehicular Environment Protocol
}

\author{
G. Jaya Suma, PhD \\ Dept. of I.T.JNTUK-University \\ College of Engineering, \\ Vizianagaram,India.
}

\author{
R.V.S. Lalitha \\ Dept.of C.S.E., \\ Sri Sai Aditya Institute of \\ Science and Technology, India.
}

\begin{abstract}
The causes of road accidents lead to emergency communication between vehicular nodes. It is true that the onthe-spot messaging helps in rescuing victim's life. The establishment of unprepared communication across vehicular nodes is possible with the intervention of automatic messaging. This leads to active research in data dissemination methods using heterogeneous devices. In this paper, the data dissemination in VANETs is done by Sensors, RFID(Radio Frequency Identification) tags, Bluetooth technology and from people passing over the roads. The communication across VANET nodes is simulated using RFID tags, the sensors available in the Android mobiles and the Bluetooth available in Android mobiles. This provides ease in communication across VANET nodes. The Web Server transmits this information to VANET nodes using WAVE(Wireless Access Vehicular Environment) protocol. The data collection and transmission across VANET is through Web Server(Middleware). Since collection of data is from different sources, Web Server plays crucial role in reception and transmission of messages across VANET nodes. In this paper, the communication methodology is optimized using $\mathrm{ABC}$ (Artificial Bee Colony) algorithm and Ant Colony algorithm. The gain of the solution is cross-verified using Least Square Fitting method and Polynomial regression for evaluating the accuracy of transmission of messages by different means.
\end{abstract}

\section{Keywords}

IEEE 802.11p, WAVE, VANET, Sensor, RFID, GSM, Least Square Fitting Model, Polynomial Regression.

\section{INTRODUCTION}

The means of communication across VANET is wireless and can be from different sources. The sources of information in this paper is from sensors, RFID(Radio Frequency Identification) and from Bluetooth. The wireless LAN allows mobile user to connect to LAN with a radio connection. Wireless LANs follow the standards of IEEE 802.11 which allows radio wave communication. Apart from the communication media the network uses hubs, bridges, routers, NIC(Network Interface Card)s and modems to connect to other networks. IEEE 802.3 LANs uses HTTP protocol and IEEE 802.11 uses wi-fi protocol. IEEE 802.11 Protocols for connecting various types of networks are: IEEE $802.3-$ Ethernet,IEEE 802.3d - Fibre optic network, IEEE 802.11 for wireless LANs, IEEE 802.11a - OFDMA(Orthogonal Frequency Division Multiple Access),IEEE 802.11p - for VANET,IEEE 1609- WAVE(Wireless Access in Vehicular Environments)[8],IEEE 802.15 - for Personnel Area Network protocols,IEEE 802.15.1 - for Bluetooth, IEEE 802.15.4 - for Zig Bee protocol, IEEE 802.15.6 - for WiMax. Smart Phone
Ad hoc Networks(SPAN) are used to exchange data between existing hardware and Bluetooth or Wi-Fi to create peer-topeer network without relying on cellular networks or network infrastructure[12]. WAP (Wireless Application Protocol) supports mobility specific enhancements Internet protocols. It sets up sets up frame work for wireless and mobile web access using GSM, GPRS, UMTS. WAP(Wireless Application Protocol) brings diverse internet content and data services into mobile. Though these are interoperable, scalable, efficient and reliable, customized applications provide ease in access with the other nodes. Also, monitoring of data can be efficiently performed. Hencetoforth, in this paper, WAE(Wireless Application Environment) is implemented using Android Sensor programming and NFC programming. GCM(Google Cloud Messaging) services, which allow mobile users to send data from server to Android applications or chrome apps. The GCM components involved in messaging are:GCM Connection servers are used to send messages between client app and Web Server, Client App(GCM enabled) is used to communicate with Web server, Web Server is the middleware component used to communicate with Client App through GCM server. Proximity Sensor is a sensor used to sense nearby object without any physical contact. Electromagnetic radiation(eg.Infrared) is used to sense object. The range that the sensor[5] can range is called nominal range. Proximity sensors are highly reliable. Proximity sensors in Smart Phones are to used to perform actions on user defined and predefined applications when it is touched. The main features of sensors are noncontact sensing, noncontact output, detection of target object even in typical environments and high response. In this paper, proximity sensors in the Android mobiles are used for simulation. Ant-Colony Algorithm[7] optimization algorithm is used to optimize the computations for displaying re-route map on the user mobile. Ultrasonic Proximity sensors use piezoelectric transducer to send and detect sound waves. This type of transducers generates high frequency sound waves to detect and reflecting off the target. Significance of Proximity Sensors is of their no physical contact to detect target object, operating at unlimited frequencies, can work even at adverse weather conditions, high range of sensing distance and can detect liquid, solid, powder state and granular objects. RFID tags are used to provide unique identifier for the object. It serves the purpose similar to bar code or magnetic strip on the ATM card. RFID tag is scanned to retrieve identification information. RFID tag[6] contains chip and small antenna. Antenna is used to receive signals from scanner(reader device), a transceiver to interpret data and a transponder(RFID tag) programmed with information. The chip can store 2,000 bytes of data. The preliminary usage is Inventory control, Library Management System and for animal security. RFID tags are of two types. 1. Active RFID tags. 2. Passive RFID tags. Active RFID tags have their own 
source and battery powered. Hence, they are always active. That means continuous emission of radio waves exists between Antenna and reader. In contrast to this Passive RFID tags are identified only when they receive signals(eg. Toll gate). The two problems that arise with RFID tags are Reader collision and Tag collision. Reader collision occurs when signals from two or more readers overlap. Tag collision occurs when many tags are present in the small area. In this paper, RFID tags communicates with the Web Server using NFC(Near Field Communication) in the Android mobile. Geocasting refers to the process of transmission of messages to destination nodes by identifying their geographical locations. Geographic destination address is identified by point/polygon/circle with center point and radius. Geographic router is the service unit that computes the distance between the source and destination by the network attached to it. In this paper, Geographic locations are obtained by using GPS(Geographic Positioning System) in the Android mobile. Intelligent Transportation Systems(ITS) includes safety related, real-time, local and situation based services such as speed limit information, intersection safety, traffic jam accident warning etc.[1]. ITS communication[9] environment is classified into wired or wireless, out of which wireless mainly take IEEE $802.11 \mathrm{p}$ as the standard[2]. Apart from that the process of communication [11] is established using Android mobiles based on Geographic Positioning System of the VANET nodes. The proposed solution deals in determining the accuracy of the solution using Least Squares Fitting method and Polynomial regression. This paper is organized as follows: Section 2 discusses related work carried out so far. The novel approach to establish communication across VANET nodes using WAVE is discussed in Section 3. Determining accuracy of the proposed methods using Least Squares Fitting and Polynomial regression is discussed in Section 4. Finally, Section 5 discusses the conclusion.

\section{RELATED WORK}

Abhijit Sarma designed HetNet(Heterogeneous Network) which is important concept for next generation wireless internet architecture and IoT(Internet of Things) where several wireless technologies coexist and users can connect to any of the technologies. IEEE802.11(WiFi) and IEEE 802.16(WiMax) are the primary technologies for building HetNets. In HetNet WiFi-WiMax integration issues are analyzed[3]. A group of overlapping APs creates WiFi hotspot under WiMAX coverage area. There can be more than one WiFi hotspots under WiMAX BS. Every MN has atleast one WiFi and one WiMAX interface. This mechanism provides proper handover privacy. Claudia Campolo discussed various issues related to service access and provisioning during multi channel operations envisioned by IEEE $802.11 \mathrm{p} / \mathrm{WAVE}$ access in VANETs. This addresses the trade off between connectivity and easiness of deployment is integrated[4]. This has been done to improve connectivity and data delivery performance[10] to decrease deployment costs. A RSU is a gateway to VANET, used to connect vehicle to Internet. This acts as a gateway to VANET traffic. Vehicles need to be equipped with two different wireless communications technologies: $1.3 \mathrm{G}$ - to provide Internet access, 2. WLAN - to provide opportunistic access through a multi-hop VANET whenever possible[13]. Khalid Adbel Hafeez proposed a novel protocol in which node dynamically formed into clusters. Cluster heads are selected based on their availability on roads and stability. The MAC protocol assists driver in providing information about driver's behavior on roads and future position. The protocol COMAC(Cluster based MAC protocol) is used to form clusters are formed based on uniqueID, speed, acceleration and direction from internal sensor network and its position from GPS[14].

\section{METHODOLOGY}

The novel approach to establish communication across VANET nodes using WAVE is demarcated in Fig.1. When an incident occurs, the messages can be from Bluetooth, RFID tag, Sensor or from a VANET node. These messages will be sent to Web Server for transmitting over the network. The messages from Sensors and RFID are programmed using Android Sensor programming and NFC programming using Android mobiles. The messages received from the Bluetooth are programmed using MIT App Inventor tool kit. Sensors and Bluetooth devices used for Android programming are from Android mobile itself. RFID tags are used for NFC communication.

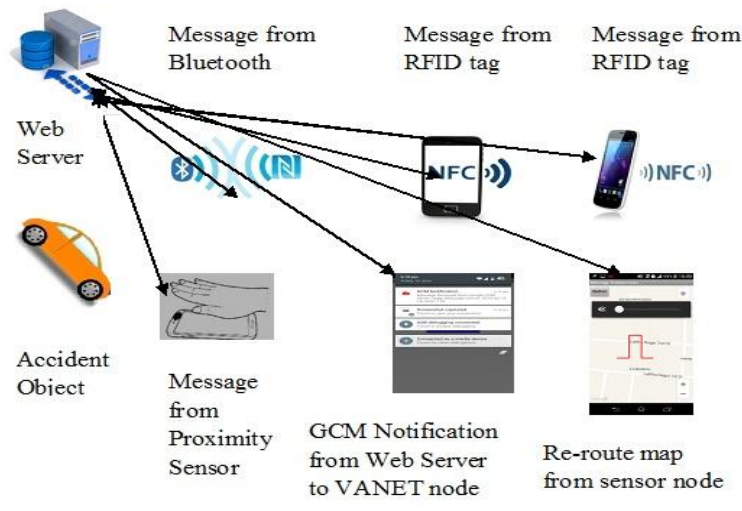

Fig. 1. VANET communication across sensors, RFID and Bluetooth using WAVE.

The message reception by the Web Server is from three different sources: 1 . from proximity sensors available in the android mobiles(e-sensor communication), 2. from RFID tags detected near by using NFC(Near Field Communication) option enabled from the Android mobiles and 3. By enabling Bluetooth using Android App.

\section{Message from proximity sensor to Web Server}

In this paper, the task is simulated using Android Sensor programming. Transmission of Emergency messages through Web Server using Google Cloud Messaging (GCM) service, which allows transmission to nearby Android mobiles (range based). The following procedure is adopted to send information to VANET users about the accident object:

1. Track latitude and longitude positions of the VANET users using GPS services.

2. Update the Location Information of the VANET users after every 20 secs using AsyncTask that runs in background as VANET is dynamic in nature.

3. Send updated coordinates along with the device id which is obtained using GCM service to server for storing in a local database.

4. Use Google Cloud Messaging service to send message to Android-powered devices using Google Map APIs.

Detection of accident objects using Proximity sensors and sending geographic location using GCM service to GCM server

As the name suggests, e-Sensor Communication uses Proximity Sensors to identify the Accident Object and sends 
alert message to Web Server for transmitting it over VANET using GCM. As soon as the Accident Object is identified by sensors available in mobile, they send the message automatically to GCM Server without intervention of the user. This reduces latency and delay in transmission. The steps involved in sensing accident object by the built in proximity sensor available in android mobile:

1. Activation of proximity sensor in the android mobile using SensorManager class via getSystemService (SENSOR_SERVICE).

2. Use proximity sensor via getDefaultSensor (TYPE_PROXIMITY) to identify the accident object.

3. The Above two play key role in identifying the accident object. As soon the object is sensed automatic messaging is to be done to the nearby users using sensors through GCM server.

4. GCM server in turn transmits this information to android-powered devices.

Providing route map to cross over the point of incident, by computing shortest distance using Ant Colony optimization algorithm

The establishment of dynamic path is computed using AntColony optimization algorithm. The algorithm for computing shortest path is as follows:

\section{Algorithm: To compute Shortest path}

Select arbitrary point near by accident object

\section{Do While}

\section{Do Until}

Choose all the possible paths from the current point to cross over the accident object by choosing arbitrary points randomly

\section{End do}

Analyze the shortest path among all possible paths

Update the Location Information based on the density of the traffic

\section{End do}

The shortest path obtained by applying the above algorithm is then displayed on the Google Map by coding. The geographic information about the location of the incident happened is tracked using Location class and sent to the server(Fig.2). The rerouting is computed using Ant-Colony optimization algorithm by finding the neighborhood latitude and longitude positions by comparing the positions to the left and the positions to the right. Thereby shortest path is decided and sent to the mobile. Finally, new reestablished path is drawn on the Google Map using Polyline class. This map is sent to the VANET user using GCM Notifications. The route map shown in Fig.3. is computed as follows. Procedure to compute Geographic positions for the reroute map in the Web server:

- Obtain Location Information of the current and arbitrary points using GPS

1. Compute Geographic distance between two points using Great Circle formula:

Distance $=\mathrm{R} * \operatorname{arcos}([\sin ($ lat 1$) * \sin ($ lat 2$)]+\cos ($ lat 1$) * \cos ($ lat $\left.2)^{*} \cos (\operatorname{lon} 2-\operatorname{lon} 1)\right]$

As the built-in proximity sensors of the mobile are used, it is assumed that current position identified by the sensor will be the accident object locations.

- To cross over the accident region, Ant-Colony optimization algorithm is used to compute shortest path dynamically. The procedure is as follows:-

Step 1:

Assumptions:

size of the object:0002km

lt1,lo1 are latitude and longitude positions.

$\mathrm{s} 1, \mathrm{~s} 2$ are the random positions chosen on the top/bottom sides.

$\mathrm{x} 1$ :lies to the left of current point

$\mathrm{x} 2$ :lies to the right of current point

$\mathrm{x} 3$ : lies to the right of $\mathrm{x} 2$

$\mathrm{v} 1$ :lies to the top of current point

v2:lies to the top of $x 2$

v3:lies to the bottom of current point

$\mathrm{v} 4:$ lies to the bottom of $\mathrm{x} 2$

Step 2:

Obtain the left/right positions by adding/subtracting the accident object size.

Obtain the top/bottom positions by adding/subtracting the random numbers computed

Current Point c(2,2) Point v1(2,5)

Point $\mathrm{x} 1(0,2) \quad$ Point v2(4,5)

Point $\mathrm{x} 2(4,2) \quad$ Point $\mathrm{v} 3(2,1)$

Point $\mathrm{x} 3(6,2) \quad$ Point $\mathrm{v} 4(4,1)$

$\operatorname{Dist} 1=\operatorname{dist}(\mathrm{x} 1, \mathrm{c})+\operatorname{dist}(\mathrm{c}, \mathrm{v} 1)+\operatorname{dist}(\mathrm{v} 1, \mathrm{v} 2)+\operatorname{dist}(\mathrm{v} 2, \mathrm{x} 2)+\operatorname{dist}(\mathrm{x} 2, \mathrm{x}$ 3)

$\operatorname{Dist} 2=\operatorname{dist}(\mathrm{x} 1, \mathrm{c})+\operatorname{dist}(\mathrm{c}, \mathrm{v} 3)+\operatorname{dist}(\mathrm{v} 3, \mathrm{v} 4)+\operatorname{dist}(\mathrm{v} 4, \mathrm{x} 2)+\operatorname{dist}(\mathrm{x} 2, \mathrm{x}$ 3)

Optimal Path=min(Dist1,Dist2)

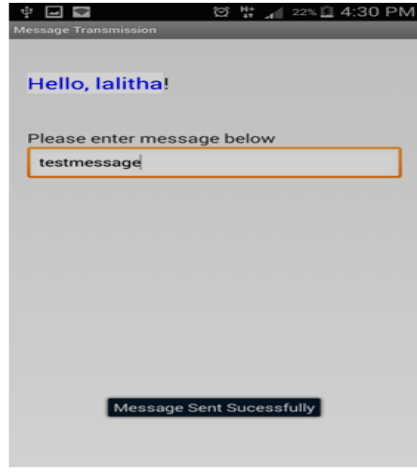

Fig.2. Sending messages to Web Server using Proximity sensors in the Android mohile

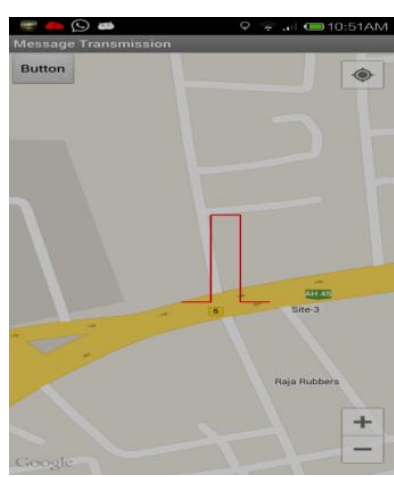

Fig.3. Displaying re-route map by the Web Server on the user's mobile. 
2. Message from RFID tag to Web Server

The steps involved in establishing communication using RFID tags are as follows:-

1. Import android.nfe package for importing RFID related classes and methods associated with them.

2. The android.nfc. NfcAdapter creates adapter between android application and RFID tag.

3. The android.nfc.NdefRecord reads data from RFID tag in terms of bytes.

4. The NfcAdapter invokes NdefMessage whenever RFID tag is discovered.

5. The setNdefPushMessage method of NfcAdapter pushes the NdefMessage messages to the display device.

Fig.4. shows the simulation of reading data from RFID tag and Fig.5. shows how GCM notifications are received into user's mobile.
Fig.4. Sending message using RFID tags

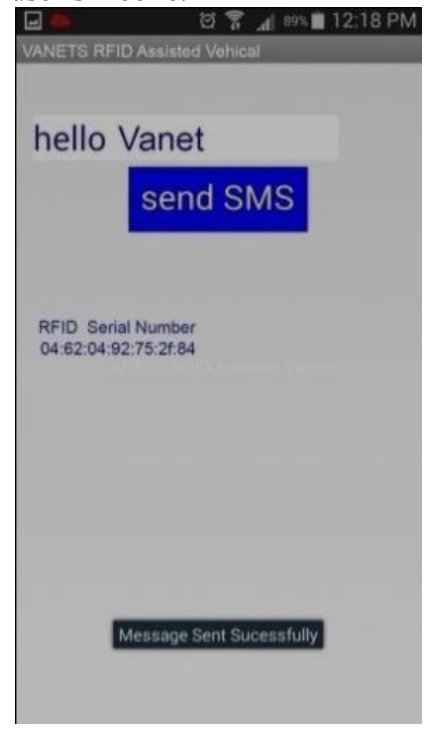

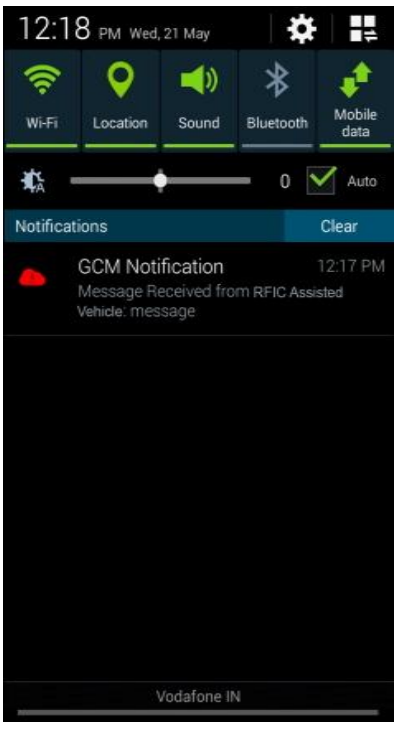

Fig. 5. Message trasmission by the Web Server as GCM notifications
3. Message from Bluetooth enabled device to Web Server

The simulation is done using MIT App Inventor tool. In this communication is established only if the nodes are paired as with normal Bluetooth communication. This provides accuracy of the information as the information is very nearer.

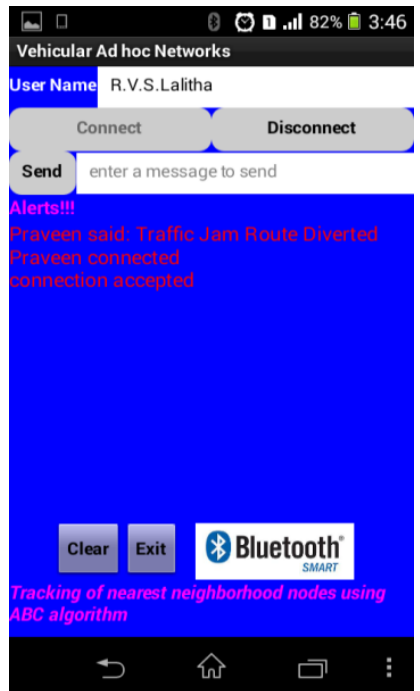

Fig.6. Message

communication by accessing Bluetooth from the Android

App.

Solution analysis using Regression

Upon receiving the messages by the Web Server, among all which message is close to the incident happened is to be analyzed. This is computed by finding the coefficient of determination which is the sum of squares of all errors $\left(R^{2}\right)$. $\mathrm{R}^{2}$ can also be represented as percentage of variation in the dependent variable to the percentage of variation in the independent variable. Hence to forth, this gives abrupt results in tracing the tests for accuracy. The point $(a, b)$ on the regression line can be computed as given below:

$\mathrm{R}^{2}$ is sum of squares of all the errors.

Condition for $\mathrm{R} 2$ to be minimum is

$\partial\left(\mathrm{R}^{2}\right) / \partial \mathrm{a}_{\mathrm{i}}=0$

Finding sum of squares of vertical deviations, for $\mathrm{i}=1$..n for a linear fit,

$\mathrm{R}^{2}(\mathrm{a}, \mathrm{b})=\sum_{i=1}^{n} \quad\left(\mathrm{y}_{\mathrm{i}}-\left(\mathrm{a}_{\mathrm{i}}+\mathrm{b}_{\mathrm{i}} \mathrm{x}_{\mathrm{i}}\right)^{2}\right.$

$\partial\left(\mathrm{R}^{2}\right) / \partial \mathrm{a}=-2 \sum_{i=1}^{n}\left[\left(\mathrm{y}_{\mathrm{i}}-\left(\mathrm{a}_{\mathrm{i}}+\mathrm{b}_{\mathrm{i}} \mathrm{x}_{\mathrm{i}}\right)\right]=0\right.$

$\partial\left(\mathrm{R}^{2}\right) / \partial \mathrm{b}=-2 \sum_{i=1}^{n}\left[\left(\mathrm{y}_{\mathrm{i}}-\left(\mathrm{a}_{\mathrm{i}}+\mathrm{b}_{\mathrm{i}} \mathrm{x}_{\mathrm{i}}\right)\right] \mathrm{x}_{\mathrm{i}}=0\right.$

These lead to the equations,

$\mathrm{na}+\mathrm{b} \sum_{i=1}^{n} \mathrm{x}_{\mathrm{i}}=\sum_{i=1}^{n} \mathrm{y}_{\mathrm{i}}$

a $\sum_{i=1}^{n} \mathrm{x}_{\mathrm{i}}+\sum_{i=1}^{n} \mathrm{x}_{\mathrm{i}}^{2}=\sum_{i=1}^{n} \mathrm{x}_{\mathrm{i}} \mathrm{y}_{\mathrm{i}}$

$\mathrm{a}=\left[\begin{array}{llllll}\sum_{i=1}^{n} & \mathrm{y}_{\mathrm{i}} & \sum_{i=1}^{n} & \mathrm{x}_{\mathrm{i}}^{2}-\sum_{i=1}^{n} \mathrm{x}_{\mathrm{i}} & \sum_{i=1}^{n} & \mathrm{x}_{\mathrm{i}} \mathrm{y}_{\mathrm{i}}\end{array}\right] /\left[\mathrm{n} \sum_{i=1}^{n} \mathrm{x}_{\mathrm{i}}{ }^{2}-\right.$ $\left.\left(\sum_{i=1}^{n} \mathrm{x}_{\mathrm{i}}\right)^{2}\right]$

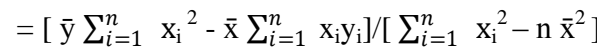

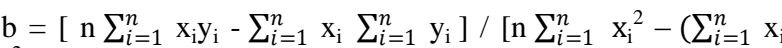
)$\left.^{2}\right]$

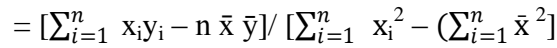

Thus $(\mathrm{a}, \mathrm{b})$ becomes data point on the regression line.

A Scenario: The Location Information of the four messages received by the Web Server are tabulated in Table 1 . 
Table 1. The Location Information of the four data points.

\begin{tabular}{|c|c|c|c|}
\hline Sl.No. & Latitude & Longitude & Location \\
\hline 1. & $16.82^{0}$ & $82.22^{0}$ & $\begin{array}{c}\text { Sarpavaram, } \\
\text { Kakinada. }\end{array}$ \\
\hline 2. & $16.98^{0}$ & $82.24^{0}$ & $\begin{array}{c}\text { JNTUK, } \\
\text { Kakinada. }\end{array}$ \\
\hline 3. & $17.05^{0}$ & 82.17 & Samalkot \\
\hline 4. & 16.72 & $81.92^{0}$ & Pithapuram \\
\hline
\end{tabular}

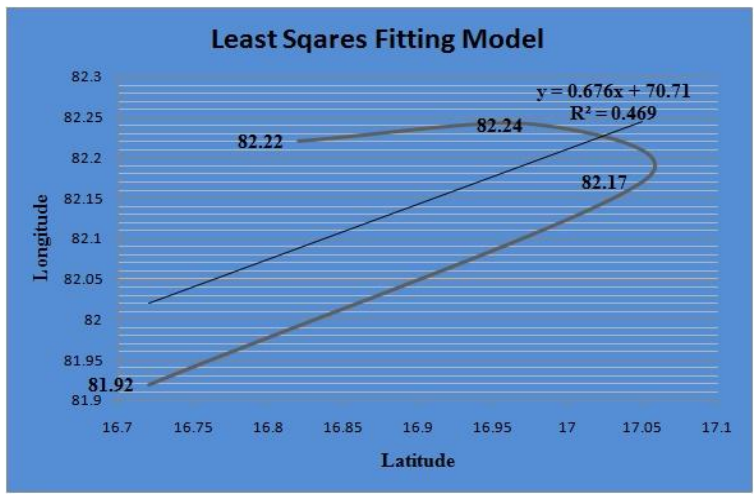

Fig.8. Graph showing the Least Square Fitting Model for the data points.

The least square error comes to 0.469 for the specified data points as shown in Fig.8. From the above analysis, it comes to true that the information from JNTUK-Kakinada(data point) and Samalkot(data point) is reliable. In addition to that, to assess the rate of accuracy among increase in no. of nodes in the VANET, Polynomial Regression is applied. Following are the computations for finding min. $\mathrm{R}^{2}$ with degree 1(Fig.9), degree 2(Fig.10) and degree 3(Fig.11).

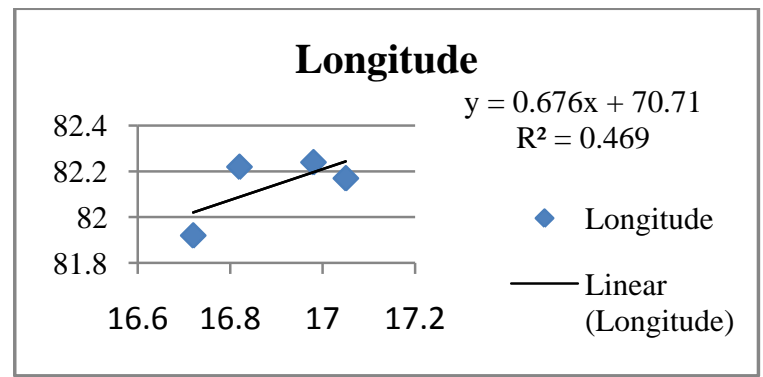

Fig.9. The min. value of $\mathrm{R} 2$ is 0.469 for Polynomial degree 1

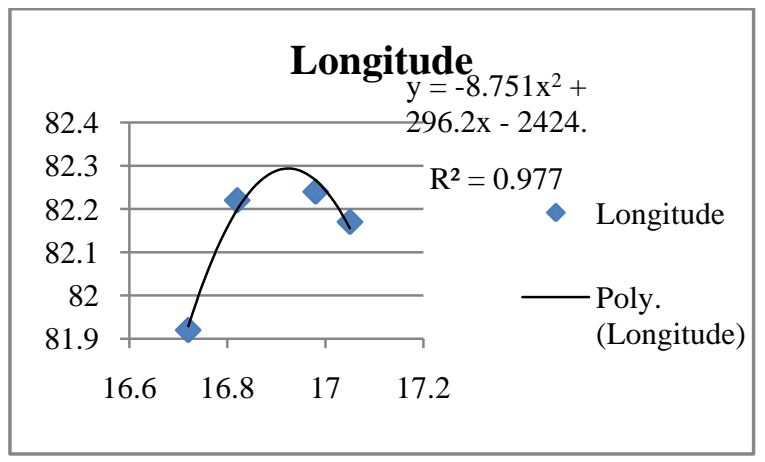

Fig.10. The min. value of $R 2$ is 0.977 for Polynomial degree 2

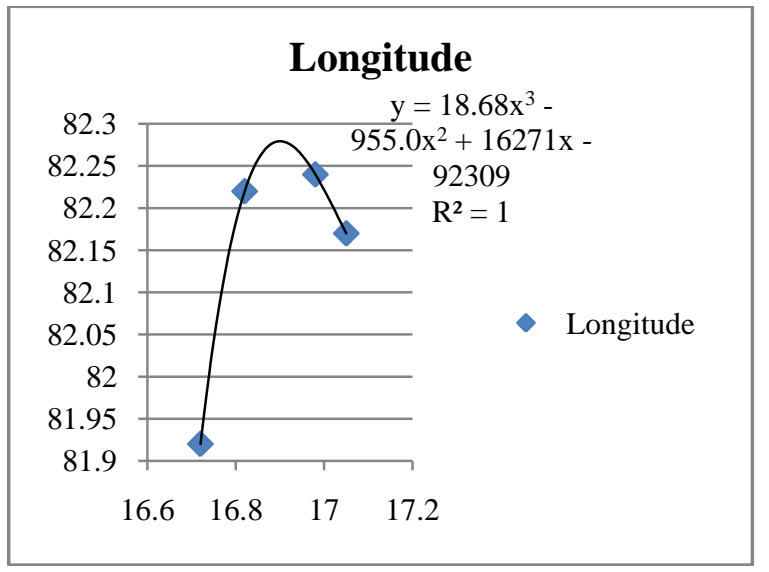

Fig.11. The min. value of $R 2$ is 1 for Polynomial degree 3

Hence, when no. of nodes is more or when the distance between the nodes is more, $\mathrm{R} 2$ value becomes maximum.

\section{CONCLUSIONS}

The automatic sensing of data fails due to its range capability, weak signal propagation and because of using low energy devices. The information captured from different devices is analyzed for testing the accuracy of the information received. This provides elegant solution in assessing the information, before taking remedial steps.

\section{REFERENCES}

[1] Vettah PathayapurayilHarigovindan, AnchareV Babu andLillykuttyJacob,"EnsuringfairaccessinIEEE801.11pb asedvehicletoinfrastructurenetworks", EURASIP Journal onWirelessCommunicationsandNetworking,2012,2012:1 68 (O)SpringerOpen Journal.

[2] Hsia-Hung Ou,Min-Shiang Hwang and Jinn-Ke Jan,"The UMTS-AKA Protocols for Intelligent Transpotation Systems", EURASIP Journal on Wireless Communications and Networking,Volume 2009,Article ID 267283,12 pages doi:10.1155/2009/267283 (C)SpringerOpen Journal.

[3] Abhijit Sarma, Member, IEEE, Sandip Chakraborty, Member, IEEE, Sukumar Nandi, Senior Member, IEEE, "Deciding Handover Points based on Context Aware Load Balancing in a WiFi-WiMAX Heterogeneous Network Environment", IEEE Transactions on Vehicular Technology, DOI.10.1109/TVT.2015.2394371 (2015).

[4] Claudia Campolo,Hector Agustin Cozzetti,Antonella Molinaro and Riccardo Scopingno,"Vehicular connectivity in urban scenarios:effectiveness and potential of roadside, moving WAVE providers and hybrid solutions", EURASIP Journal on Wireless Communications and Networking,2011,2011:146 (C)SpringerOpen Journal.

[5] Shinya Toyonga, Daichi Kominami, Masashi Sugano and Masayuki Murta, " Potential-based routing for supporting robuts any-to-any communication in wireless sensor networks',EURASIP Journal on Wireless Communications and Networking,2013,2013:278@SpringerOpen Journal.

[6] Eun-Kyu-Lee, "RF-GPS : RFID Assisted Localization in VANETs", Department of Computer Science, University of California,IEEE,2009.

[7] Parpinelli R., Lopes H., Freitas A.:Data Mining with an 
Ant Colony Optimization Algorithm, Vol 5217, LNCS,Springer,2008.

[8] Subir Biswas, Member, IEEE, and Jelena Misic, Senior Member, IEEE, "A Cross-Layer Approach to PrivacyPreserving Authentication in WAVE-Enabled VANETs", IEEE Transactions on Vehicular Technology, Vol.62, No.5, June 2013 (2013).

[9] Panayiotis Kolios, Christos Panayiotou, and Georgios Ellinas, "ExTraCT: Expediting Offloading Transfers Through Intervehicle Communication Transmissions", IEEE Transactions on Intelligent Transportation Systems (2014)

[10] Manuel Fogue, Piedad Garrido, Member, IEEE, Francisco J. Martinez, Member, IEEE, Juan-Carlos Cano, Carlos T. Calafate, and Pietro Manzoni, Member, IEEE, "A System for Automatic Notification and Severity Estimation of Automotive Accidents", IEEE Transactions on Mobile Computing, Vol.13, No.5, May 2014 (2014).
[11] Hamid Reza Arkian, Reza Ebrahimi Atani, Atefe Pourkhalili, Saman Kamali, "Cluster-based traffic information generalization in Vehicular Ad-hoc Networks", Department of Computer Engineering, University of Guilan, Rasht, Iran, Vehicular Communications, Elsevier (2014)

[12] Joao A. F. F. Dias, Joel J. P. C Rodrigues, Liang Zhou, "Coopeartion advances on vehicular communications: A survey”, Vehicular Communications, Elsevier (2014).

[13] Marco Gramaglia, Carlos J Bernardos and Maria Calderon,"Seamless internet 3G opportunistic WLAN vehicular connectivity", EURASIP Journal on Wireless Communications and Networking,2011,2011:183 (C)SpringerOpen Journal.

[14] Khalid Abdel Hafeez,Lian Zhao Liao and Bobby NgokWah Ma,"Clustering and OFDMA-based MAC protocol(COMAC) for vehicular ad hoc networks", EURASIP Journal on Wireless Communications and Networking,2011,2011:117 @SpringerOpen Journal. 